\title{
Fuzzy Logic Adaptive Mobile Location Estimation
}

\author{
Jongchan Lee ${ }^{1}$, Seung-Jae Yoo $^{2}$, Dong Chun Lee ${ }^{3}$ \\ ${ }^{1}$ Radio Access Research Team ETRI, Korea \\ chan2000@etri.re.kr \\ ${ }^{2}$ Dept. of Information Security Joongbu Univ., Korea \\ ${ }^{3}$ Dept. of Computer Science, Howon Univ., Korea
}

\begin{abstract}
In this study, we propose a novel mobile tracking scheme which utilizes the fuzzy-based decision making with the consideration of the information such as previous location, moving direction and distance to the base station as well as received signal strength, thereby resulting to the estimation performance even much better than the previous schemes. Our scheme divides a cell into many blocks based on the signal strength and then estimate in stepwise the optimal block where a mobile locates using MultiCriteria Decision Making (MCDM). Through numerical results, we show that our proposed mobile tracking method provides a better performance than the conventional method using the received signal strength.
\end{abstract}

\section{Introduction}

In the microcell-based or picocell-based mobile communication network frequent movements of a mobile terminal or host bring about excessive traffics into the network and may degrade the quality of services (QoS) severely. If its location can be estimated, network resources may be more effectively allocated and better QoS can be provisioned with the combination of handoff optimization. Moreover the location estimation technology may be used in other new applications such as the emergency call for disaster recovery. It will have viable roles in the communication networks of next generation. Global Positioning System (GPS) was initially developed for military purposes but it is also utilized for civil applications such as local traffic information services and geo-location based applications. However incorporating GPS receivers into handsets raises questions of cost, size and power consumption [1].

Other methods for location estimation are based on radio signal propagation such as signposts, dead reckoning, circular or hyperbolic trilateration systems, etc. Many methods and systems have been proposed based on radio signal strength measurement of a mobile object's transmitter by a set of base stations [2,3,4]. Recently, adaptive schemes based on the use of cellular systems and on fuzzy logic [5], hidden Markov models $[6,7]$ and pattern recognition methods [8] have been used to estimate the position of mobiles. The system studied in [2] estimates mobile location using information on contours but it does not provide a realistic search procedure. In [3] the estimation is based on the signal strength received at a multi-beam antenna of a base station in the multi-path environment, and the angle of its arrival (AOA). AOA is measured under the assumption that the signal is in line of sight (LOS), but LOS 
signal may not be received in the microcell where reflections and diffractions occur due to dense building environment. In this situation AOA of the strongest reflected signal is utilized for estimation, and therefore the location estimated differs greatly from real one. Time of arrival (TOA) of a signal from a mobile to neighboring base stations are used in [9], but this scheme has two problems. First, an accurate synchronization is essential between all sending endpoints and all receiving ones in the system. An error of $1 \mu \mathrm{s}$ in synchronization results to $300 \mathrm{~m}$ error in location. Secondly this scheme is not suitable for the microcellular environment because it also assumes LOS environment. Time difference of arrival (TDOA) of signals from two base stations is considered in [10]. TOA scheme and TDOA scheme have been studied for IS-95B where PN code of CDMA system can be used for the location estimation. Enhanced Observed Time Difference (E-OTD) is a TDOA positioning method based on OTD feature already existing in GSM. The mobile measures arrival time of signals from three or more cell sites in a network. In this method the position of mobile is determined by trilateration [11]. E-OTD, which relies upon the visibility of at least three cell sites to calculate it, is not a good solution for rural areas where cell-site separation is large. However, it promises to work well in areas of high cellsite density and indoors.

The above-mentioned schemes such as AOA, TOA and TDOA have problems as follows.

- These schemes assume that the cellular system consists of LOS areas. They get good results only under this assumption.

- The microcellular system such as IMT-2000 has NLOS areas which are affected by specific reflections and diffractions. In this situation these schemes have great errors in estimation.

- In the microcellular environment the points of the same average signal strength form not a circular contour but a distorted one. These schemes ignore the fact that the propagation rule is affected by many parameters.

- They rely only on the information related to radio signal such as signal strength. Their accuracies are affected by short-term fading, shadowing or diffraction.

In this study, to enhance estimation accuracy, we propose a scheme based on MultiCriteria Decision Making (MCDM) which considers multiple parameters: the signal strength, the distance between the base station and mobile, the moving direction, and the previous location. This process is based on three step location estimations which can determine the mobile position by gradually reducing the area of the mobile position [12]. Using MCDM, the estimator first estimates the locating sector in the sector estimation step, then estimates the locating zone in the zone estimation step, and then finally estimates the locating block in the block estimate step.

\section{Estimation Procedure}

Figure 1 shows how our scheme divides a cell into many blocks based on the signal strength and then estimates the optimal block stepwise where the mobile is located using MCDM. 


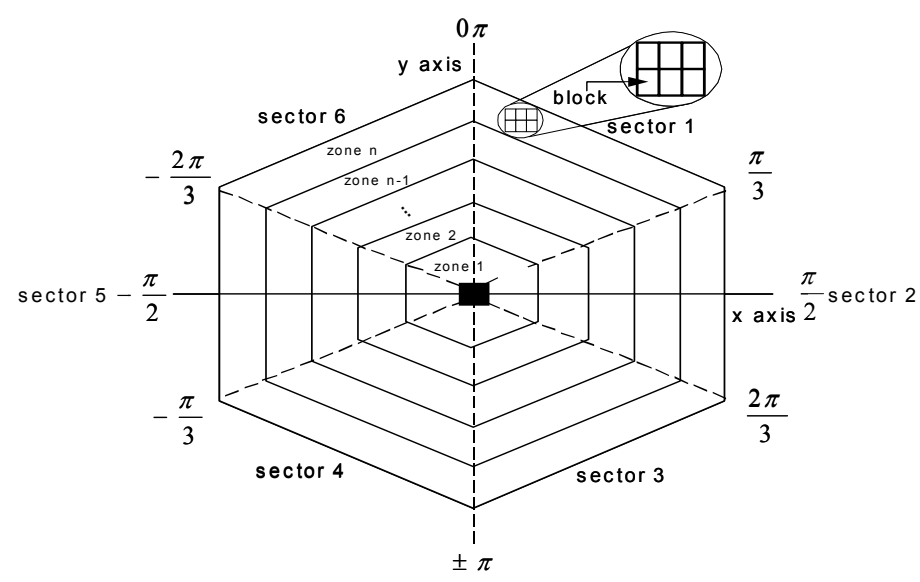

Fig. 1. Sector, Zone and Block

The location of a mobile within a cell can be defined by dividing each cell into sectors, zones and blocks and relating these to the signal level received by it at that point. It is done automatically in three phases of sector definition, zone definition and block definition. Then the location definition block is constructed with these results. They are performed at the system initialization before executing the location estimation. The sector definition phase divides a cell into sectors, and assigns a sector number to blocks belonging to each sector. The zone definition phase divides each sector into zones, and assigns a zone number to blocks belonging to each zone. The block definition phase assigns a block number to each block. In order to indicate the location of each block within a cell, 2-dimensional vector $(d, a)$ is assigned to each block. After the completion of this phase each block has a set of block information.

The collection of block information is called the block object. The block object contains the following information: the sector number, the zone number, the block number, the vector data $(\mathrm{d}, \mathrm{a})$, the maximum and the minimum value of average PSS for the LOS block, the compensated value for the NLOS block and a bit for indicating "node" or "edge", etc.

Using MCDM and the block object which is constructed as described above, the estimator is started with a timer, and the estimation is performed sequentially in three steps: sector estimation, zone estimation, and finally block estimation.

\section{Mobile Tracking Based on MCDM}

\subsection{Multi-Criteria Decision Parameters}

In our study, the received signal strength, the distance between the mobile and the base station, the previous location, and the moving direction are considered as decision parameters. The received signal strength has been used in many schemes, but it has very irregular profiles due to the effects of radio environments. The distance is 
considered because it can explain the block allocation plan; however, it may also be inaccurate due to the effect of multi-path fading, etc. It is not sufficient by itself. We consider the previous location. It is normally expected that the estimated location should be near the previous one. Therefore, if the estimated location is too far from the previous one, the estimation may be regarded as inaccurate. We also consider the moving direction. Usually the mobile is most likely to move forward, less likely to move rightward or leftward, and least likely to move backward more than one block. The low-speed mobile (a pedestrian) has a smaller moving radius and a more complex moving pattern, while the high-speed mobile (a motor vehicle) has a larger radius and a simpler pattern.

In mobile tracking using MCDM, the Decision function $D$ is defined by combining the degree of satisfaction for multiple evaluation parameters, and the decision is made on the basis of his function. The evaluation parameter can be seen as a proposition. A compound proposition is formed from multiple evaluation parameters with a connective operator, and the total evaluation is performed by totaling the values for the multiple parameters with connective operators. In this method errors in the evaluation parameters impose milder changes on the total evaluation value than in binary logics.

\subsection{Membership Function}

The membership function with a trapezoidal shape is used for determining the membership degree of the mobile because it provides a more versatile degree between the upper limit and the lower one than the membership function with a step-like shape. Let us define the membership functions for the pilot signal strengths from neighboring base stations.

The membership function of $P S S_{i}, \mu_{R}\left(P S S_{i}\right)$, is given by Figure $2 . P S S_{i}$ is the signal strength received from the base station $i, s_{1}$ is the lower limit, and $s_{2}$ is the upper limit.

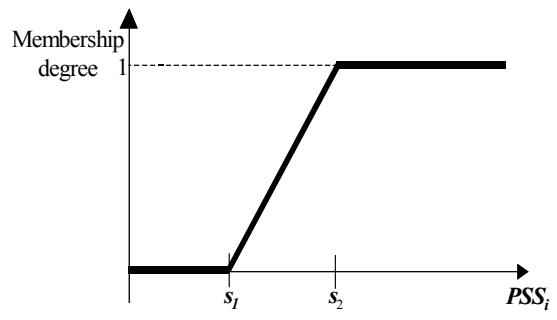

Fig. 2. The membership function of the PPS.

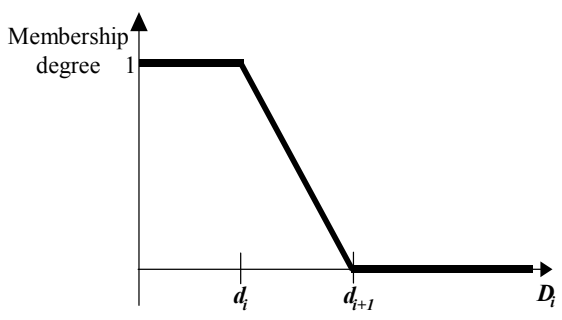

Fig. 3. The membership function of the distance

Now we define the membership function of the distance. The membership function of the distance, $\mu_{R}\left(D_{i}\right)$, is given by Figure 3, where $D_{i}$ is the distance between the base station $i$ and the mobile, $d_{1}$ is the upper limit, and $d_{2}$ is the lower limit. 
The membership function of the previous location of the mobile, $\mu_{R}\left(L_{i}\right)$, is given by Figure 4. Where $L_{i}$ is the vector information of its current location, $E_{1}, \cdots, E_{4}$ is the vector information of the previous location, and $g_{i}$ is the physical difference between them.

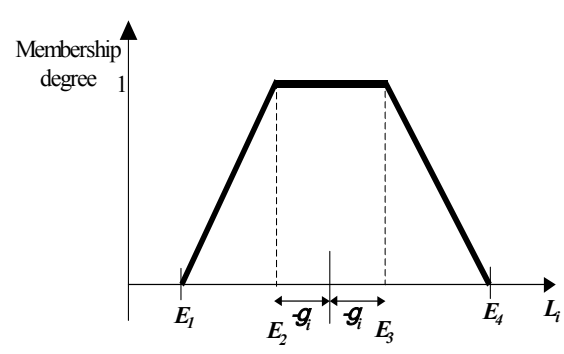

Fig. 4. The membership function of the location.

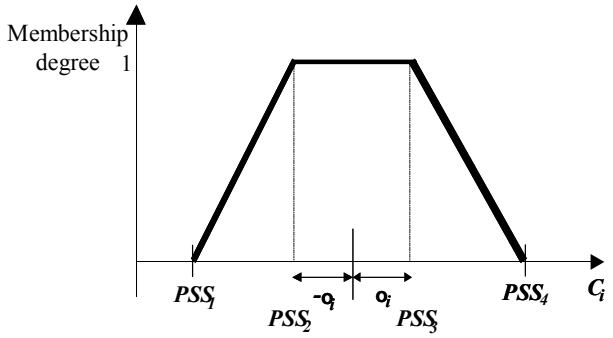

Fig. 5. The membership function of the direction.

The membership function of the moving direction, $\mu_{R}\left(C_{i}\right)$, is given by Figure 5. $C_{i}$ is the vector information of the moving direction, $P S S_{1}, \cdots, P S S_{4}$ is the pilot signal strength and $o_{i}$ the physical difference between the previous location and the current one

\subsection{Location Estimation}

Most of the MCDM approaches face the decision problem in two consecutive steps: aggregating all the judgments with respect to all the criteria and per decision alternative and ranking the alternatives according to the aggregated criterion. Also our approach uses this two-steps decomposition [13].

Let $J_{i}(\mathrm{i} \in\{1,2, \ldots, \mathrm{n}\}$ be a finite number of alternatives to be evaluated against a set of criteria $K_{j}(\mathrm{j}=1,2, \ldots, \mathrm{m})$. Subjective assessments are to be given to determine (a) the degree to which each alternative satisfies each criterion, represented as a fuzzy matrix referred to as the decision matrix, and (b) how important each criterion is for the problem evaluated, represented as a fuzzy vector referred to as the weighting vector.

Each decision problem involves $n$ alternatives and $m$ linguistic attributes corresponding to $m$ criteria. Thus, decision data can be organized in a $m \times n$ matrix. The decision matrix for alternatives is given by Eq. (1):

$$
\mu=\left[\begin{array}{cccc}
\mu_{R}\left(P S S_{11}\right) & \mu_{R}\left(D_{12}\right) & \mu_{R}\left(L_{13}\right) & \mu_{R}\left(C_{14}\right) \\
\mu_{R}\left(P S S_{21}\right) & \mu_{R}\left(D_{12}\right) & \mu_{R}\left(L_{13}\right) & \mu_{R}\left(C_{14}\right) \\
\mu_{R}\left(P S S_{31}\right) & \mu_{R}\left(D_{12}\right) & \mu_{R}\left(L_{13}\right) & \mu_{R}\left(C_{14}\right) \\
\ldots & \ldots & \ldots & \ldots \\
\mu_{R}\left(P S S_{n 1}\right) & \mu_{R}\left(D_{n 2}\right) & \mu_{R}\left(L_{n 3}\right) & \mu_{R}\left(C_{n m}\right)
\end{array}\right]
$$


The weighting vector for evaluation criteria can be given by using linguistic terminology with fuzzy set theory [14]. It is a finite set of ordered symbols to represent the weights of the criteria using the following linear ordering: very high $\geq$ high $\geq$ medium $\geq$ low $\geq$ very low. Weighting vector $W$ is represented as Eq. (2).

$$
W=\left(w_{i}^{P S S}, w_{i}^{D}, w_{i}^{L}, w_{i}^{C}\right)
$$

The fuzzification procedure leads to a performance matrix $\mu \in[0,1]^{n \times m}$ where each element $\mu_{n m}$ expresses how much the $n$-th alternative satisfies the $m$-th criterion. Therefore, each low of the performance matrix is a fuzzy set $\mu_{m}$ expressing the satisfaction of the $m$-th criterion in the universe of the available alternatives [13-14]. By multiplying the weighting vector by the decision matrix, the performance matrix is

$$
\mu=\left[\begin{array}{cccc}
\mu_{R}\left(P S S_{1}\right) \times w_{1}^{P S S} & \mu_{R}\left(D_{12}\right) \times w_{1}^{D} & \mu_{R}\left(L_{13}\right) \times w_{1}^{L} & \mu_{R}\left(C_{14}\right) \times w_{1}^{C} \\
\mu_{R}\left(P S S_{11}\right) \times w_{2}^{P S S} & \mu_{R}\left(D_{12}\right) \times w_{2}^{D} & \mu_{R}\left(L_{13}\right) \times w_{2}^{L} & \mu_{R}\left(C_{14}\right) \times w_{2}^{C} \\
\mu_{R}\left(P S S_{1}\right) \times w_{3}^{P S S} & \mu_{R}\left(D_{12}\right) \times w_{3}^{D} & \mu_{R}\left(L_{13}\right) \times w_{3}^{L} & \mu_{R}\left(C_{14}\right) \times w_{3}^{C} \\
\ldots & \ldots & \ldots & \ldots \\
\mu_{R}\left(P S S_{n 1}\right) \times w_{i}^{P S S} & \mu_{R}\left(D_{n 2}\right) \times w_{i}^{D} & \mu_{R}\left(L_{n 3}\right) \times w_{i}^{L} & \mu_{R}\left(C_{n m}\right) \times w_{i}^{C}
\end{array}\right]
$$

given by Eq. (3):

Given the decision matrix and the weighting vector, the decision making objective for the general fuzzy MCDM problem is to rank all alternatives by giving each of them an overall preference rating with respect to all criteria [14]. GMV (Generalized Mean Value) is used for ranking the alternatives according to the aggregated criterion. The GMV for alternatives is represented as Eq. (4).

$$
m\left(\mu_{n}\right)=\frac{\left(C_{i}+D_{i}\right)^{2}-\left(A_{i}+B_{i}\right)^{2}+A_{i} \cdot B_{i}-C_{i} \cdot D_{i}}{3 \cdot\left[\left(C_{i}+D_{i}\right)-\left(A_{i}+B_{i}\right)\right]}
$$

where $A_{i}=\mu_{R}\left(P S S_{n 1}\right) \times w_{i}^{P S S}, \quad B_{i}=\mu_{R}\left(D_{n 2}\right) \times w_{i}^{D}, \quad$ and $\quad C_{i}=\mu_{R}\left(L_{n 3}\right) \times w_{i}^{L}$, $D_{i}=\mu_{R}\left(C_{n m}\right) \times w_{i}^{C}$, respectively.

\section{Performance Analysis}

The moving path and the mobile velocity are affected by the road topology. The moving pattern is described by the changes in moving direction and velocity. In our study we assume that low speed mobiles, pedestrians, occupy $60 \%$ of the total population in the cell and high-speed mobiles, vehicles, $40 \%$. One half of the pedestrians are assumed to be still and another half moving. Also the private owned cars occupy $60 \%$ of the total vehicle, the taxi $10 \%$ and the public transportation $30 \%$. 
Vehicles move forward, leftward/rightward and U- Turn. The moving velocity is assumed to have a uniform distribution. The walking speed of pedestrians is $0 \sim 5$ $\mathrm{Km} / \mathrm{hr}$, the speed of private cars and taxis $30 \sim 100 \mathrm{Km} / \mathrm{hr}$, and buses $10 \sim 70 \mathrm{Km} / \mathrm{hr}$. The speed is assumed to be constant during walking or driving. Figure 4 shows the road used in our simulation to consider traffic environments. The black circle indicates the branch of the road, and the shaded areas are blocks that the road passes through. Each block is a square and its side is assumed to have the length of $30 \mathrm{~m}$. The time needed for a high speed mobile to pass through a block is calculated from $B T=r / v$ where $\mathrm{r}$ is the length of the road segment crossing at each block and $\mathrm{v}$ the mobile speed. As shown in Figure 4, BT is dependent on $\mathrm{r}$. We can consider four different values - $r, n \sqrt{2} m$ (crossing diagonally), $\frac{3 n}{4} \sqrt{2} m$ (3/4 crossing), $\frac{n}{2} \sqrt{2} m$ (2/4 crossing) and $\frac{n}{4} \sqrt{2} m$ (1/4 crossing) - according to which portion of a block each road segment crosses through. In order to reflect more realistic information into our simulation, it is assumed that the signal strength is sampled every $0.5 \mathrm{sec}, 0.2 \mathrm{sec}, 0.1$ sec, $0.1 \mathrm{sec}$ and $0.05 \mathrm{sec}$ for the speed of $\leq 10 \mathrm{~km} / \mathrm{h}, \leq 20 \mathrm{~km} / \mathrm{h}, \leq 50 \mathrm{~km} / \mathrm{h}, \leq 70 \mathrm{~km} / \mathrm{h}$ and $\leq 100 \mathrm{~km} / \mathrm{h}$, respectively. If BT is too small, we cannot obtain enough samples to calculate the average signal strength. We consider the following simulation parameters regarding the received signal strength. The value of $k_{2}$, which indicates the changes in LOS/NLOS environments, is in the range of 20 through 50 . The mean signal attenuation by the path-loss is proportional to 3.5 times the propagation distance, and the shadowing has a log-normal distribution with a standard deviation of $\sigma=6 d B$. A value of received signal strength less than $-16 d B$ is regarded as an error, which is therefore excluded from the calculation.

To evaluate the error probability of our schemes in each estimation stage, the mobile population in the track boundary and sector boundary is generated according to a Poisson distribution. All the mobiles generated above are assumed to cross the sector boundary lines and track boundary lines. Also the curved path passes through the handoff area. Stationary mobiles appear at sector boundary areas and track boundary areas and remain still at those points. Pedestrian mobiles appear at sector and track boundary areas and move toward the neighboring sector or track.

Figure 6 shows the effect of the block size on the estimation performance of MCDM and the existing schemes. As the block size becomes smaller, the accuracies of the three schemes decrease. The accuracies of AOA and TOA decrease rapidly. On the other hand, the performance of MCDM is least affected by block size because it additionally utilizes the previous location and distance between the mobile and the base station for estimation.

Figure 7 shows the estimation rate or accuracy of our proposed scheme depending on the mobile speed. The accuracy of AOA and TOA becomes lower rapidly since the signal measurement error would be large as the mobile speed increases. The performance of MCDM is least affected by the mobile speed because the information such as moving direction and previous location are considered in MCDM and, therefore, errors during the signal evaluation step decrease. We compare our scheme, MCDM with VA [12], E-OTD and TDOA in Figure 8. In this figure the mobile maintains its y position at $1000 \mathrm{~m}$ and traverse $x$ axis from $x=0 \mathrm{~m}$ to $x=2000 \mathrm{~m}$. 


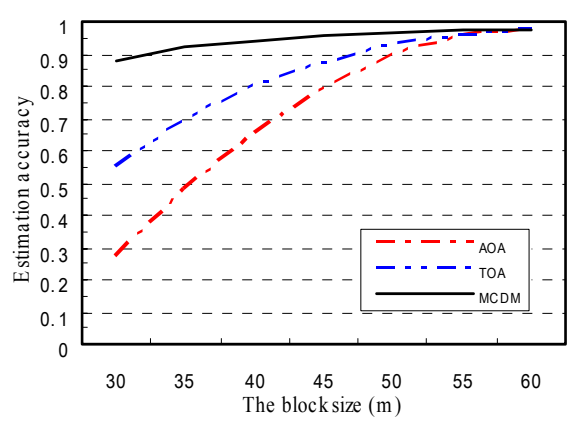

Fig. 6.The estimation accuracy versus the size

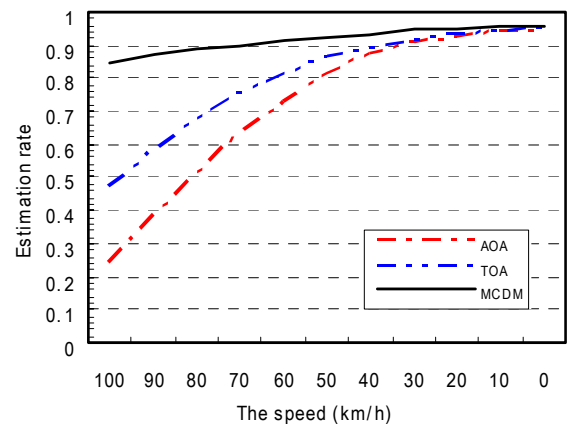

Fig. 7. The estimation accuracy versus the speed

Distance Root Mean Square stands for the root-mean-square value of the distances from the true location point of the position fixes in a collection of measurements. In order to get the estimated values for comparison we take an average of 20 values for each mobile position. We assume NLOS environment and the signal level of mobile may change abruptly due to shadowing. It shows that the performance of MCDM is least affected by abrupt change of signal level. MCDM has the most accurate result. This may well be attributed to the fact that it imposes less weight on the received signal strength in NLOS area and, instead, greater weights on other parameters such as the distance between mobile and base station, previous location, and moving direction are considered as decision parameters

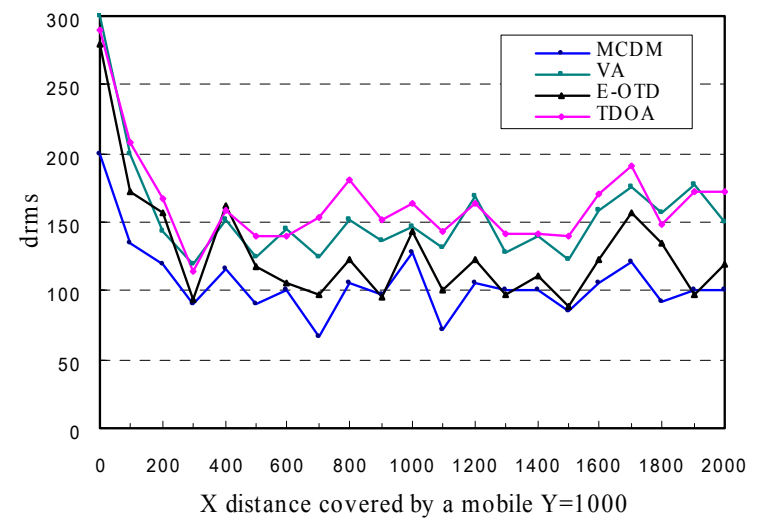

Fig. 8. Comparison of the estimation accuracy

\section{Conclusions}

In this study, we proposed a MCDM-based mobile tracking method for estimating more accurately the mobile location by considering multiple parameters, such as the signal strength, the distance between the base station and mobile, the moving 
direction, and previous location. We have demonstrated that our scheme increases the estimation accuracy when the mobile moves along a boundary area. Further, we have shown that the proposed scheme is little affected by an increased mobile speed or a decreased block size. The effect of weight factor variations on the estimation performance of our scheme and the determination of the optimal weight should be the subject of a future study.

\section{References}

[1] G. M. Djuknic and R. E. Richton, "Geolocation and Assisted GPS," IEEE Computer, Vol. 34, No. 2, pp.123-125, Feb. 2001.

[2] W. G. Figel, N. H. Shepherd and W. F. Trammell, "Vehicle location by a signal attenuation method," IEEE Trans. Veh. Technol., vol. VT-18, pp. 104-109, Nov. 1969.

[3] G. D. Ott, "Vehicle location in cellular mobile radio systems," IEEE Trans. Veh. Tech., Vol. VT-26, pp. 43-46, Feb. 1977.

[4] Hatta M., Nagatsu T., "Mobile Location Using Signal Strength Measurements in a Cellular System", IEEE Transactions on Vehicular Technology, vol. VT29, pp245 - 252, May 1980.

[5] Song H.L., "Automatic Vehicle Location in Cellular Communication Systems", IEEE Transactions on Vehicular Technology, vol.43, pp902-908, Nov. 1994.

[6] Kennemann O., "Pattern Recognition by Hidden Markov Models for Supporting Handover Decisions in the GSM system", in Proc. 6 th Nordic Seminar Dig. Mobile Radio Comm., Stockholm,Sweden, pp.195-202, 1994

[7] T. Nypan and O. Hallingstad, "Cellular Positioning by Database Comparison and Hidden Markov Models.," PWC2002:, pp. 277-284, Oct. 2002

[8] Kennemann O., "Continuous Location of Moving GSM Mobile Stations by Pattern Recognition Techniques", in Proc. 5th Int. Symp. Personal, Indoor, Mobile, Radio Comm., denHaag, Holland, pp.630-634, 1994.

[9] H. Staras and S. N. Honikman, "The accuracy of vehicle location by trilateration in a dense urban environment," IEEE Trans. Veh. Tech., vol VT-26, pp. 38-43, Feb. 1972.

[10] T. S. Rappaport, J. H. Reed and B. D. Woerner, "Position Location Using Wireless Communications on Highways of the Future," IEEE Communications Magazine, pp. 33-41, Oct.

[11] Y. A. Spirito, "On the Accuracy of Cellular Mobile Station Location Estimation," IEEE Trans. Veh. Technol., vol. 50, no. 3, pp. 674-685, 2001.

[12] J. C. Lee and Y. S. Mun, "Mobile Location Estimation Scheme," SK telecommunications Review, Vol. 9, No. 6, pp. 968-983, Dec. 1999.

[13] C. Naso and B. Turchiano, "A Fuzzy Multi-Criteria Algorithm for Dynamic Routing in FMS," IEEE ICSMC'1998, Vol. 1, pp. 457-462, Oct. 1998. 